\title{
Fe nano-particle coatings for high temperature wear resistance
}

\author{
J. C. Walker ${ }^{1 *}$, S. R. Saranu ${ }^{2}$, A. H. Kean ${ }^{2}$, R. J. K. Wood ${ }^{1}$ \\ ${ }^{I}$ National Centre for Advanced Tribology at Southampton (nCATS), Highfield Campus, Southampton, SO17 1BJ, UK \\ ${ }^{2}$ Mantis Deposition Ltd, 2 Goodson Industrial Mews, Wellington Street, Thame, Oxon, OX9 3BX, UK
}

Received Date Line (to be inserted by Production) ( $8 \mathrm{pt}$ )

\begin{abstract}
Oxidational wear continues to present an economic challenge for the replacement of components subject to high temperature fretting and sliding contacts in applications such as gas turbine engines. At elevated temperatures, low friction oxide 'glaze' layers can form and act as an interface between the contact and the substrate material. Whilst desirable, the glaze is formed from wear debris and often consumes the underlying substrate material. In order to induce rapid formation of low friction oxide layers without a severe 'running-in' period, nano particles of $\mathrm{Fe}$ in the range 5-10nm were deposited on ground flat ended pin and plate 080M40 substrates using a terminated gas condensation PVD process, to a thickness of 600nm. Coatings were tested in a reciprocating geometry at a fixed stroke length of $0.4 \mathrm{~mm}$, frequency of $31 \mathrm{~Hz}$ and $40 \mathrm{~N}$ normal load (1MPa contact stress) and at ambient, $300^{\circ} \mathrm{C}$ and $540^{\circ} \mathrm{C}$. At ambient temperature the coated surfaces exhibited higher friction but lower wear compared to the uncoated substrates, whereas at elevated temperatures, the coated surfaces exhibited slightly lower steady state dynamic friction coefficients, and minimal changes in wear depth after a short incubation period. SEM of the worn surfaces indicated that hard oxide plateaus were responsible for the load bearing contact area at elevated temperatures. Cross sectional FIB, TEM and SIMS confirmed that at elevated temperatures, the nano-particle coating induced rapid formation of a nano-crystalline porous surface oxide film of mixed composition which protected the substrate from severe wear during the running-in period.
\end{abstract}

Keywords: Fe nano-particles, Oxidation, Wear, FIB, SIMS

\section{Introduction}

Oxidational wear of metallic components subject to fretting and sliding contacts continues to be a major industrial problem in the automotive, aerospace and energy sectors where component failure can lead to expensive maintenance downtime. The phenomena of metallic oxidational wear has been studied at some length by Quinn [1-3] and others [4-6], many of whom noted that the interfacial film generated during oxidative wear can, if the

*Corresponding author. Tel.: +44 2380 592371; fax: +44 2380595167.

E-mail address:j.walker@soton.ac.uk (John Walker) 
conditions are right, be of benefit to the friction behaviour of the contact, acting as a protective intermediate tribofilm between the metallic contact surfaces. Indeed, such studies repeatedly pointed to the transition from an initially severe wear period during running-in, to a mild wear regime, based upon sliding distance, velocity and contact load.

For elevated temperature contacts, much work has been devoted to the investigation of so called compacted protective oxide layers or 'glaze' layers, which offer a low friction oxide layer of low shear strength which acts as a more desirable interfacial tribo-layer between metallic contacts [7-12]. The critical component for the formation of a such layers is the presence of nano-scale oxide debris and hence the incubation period is a process of wear debris formation, comminution and agglomeration to form compacted regions of debris on the surface, all of which takes place during the initial severe wear running-in period [13]. Once the surrounding material has been worn away, the compacted oxide regions subsequently become load bearing, acting as an interlayer between the metallic surfaces $[14,15]$. The difference between compacted oxide wear debris and 'glaze' layers is that sintering between the oxidised wear debris particles results in bonding between the particles. The wear protection offered by a 'glaze' is usually superior to a compacted oxide debris layer [16], although both tend to offer superior friction and wear resistance compared to metal-on-metal contact $[9,12,15]$. Thus for the formation of 'glaze' layers, the rate determining step is not so much the oxidation rate of the surface or the formation of wear debris, but the activation energy for adhesion/sintering of the oxidised particles [15]. This is why higher temperatures result in the more rapid formation of 'glaze' layers after sliding has commenced $[8,15]$.

One method attempted to induce the rapid formation of a beneficial oxide glaze layer was the static pre-oxidation of metallic surfaces. Whilst this has been successful at higher temperatures for Co-based coating pre-oxidised at $600^{\circ} \mathrm{C}[13]$, it appeared that breakdown of the oxide layer was still necessary for the formation of a compact nanoscale protective layer. Thus a number of authors have attempted to use external oxide particle additions to the contact surface as a means to nucleate and catalyse the formation of a wear protective oxide [17-22].

Colombie et al [22] looked at the addition of $\mathrm{Fe}_{2} \mathrm{O}_{3}$ and $\mathrm{Fe}_{3} \mathrm{O}_{4}$ particles (12 and 24 $\mu$ m median) to steel contacts under fretting conditions. They found that addition of $\mathrm{Fe}_{2} \mathrm{O}_{3}$ particles resulted in a smooth worn surface as a result of the compacted oxide. Mass losses were lower than identical tests carried out without particle addition. This concept was further explored by Iwabuchi and co-workers $[17,18]$ who looked specifically at the effect of $\alpha$ $\mathrm{Fe}_{2} \mathrm{O}_{3}$ particles on the severe-mild wear transition in S45C steel. They found that addition of these particles led to a transition in the severe to mild wear rate and that smaller particles seemed to cause a faster transition. It also

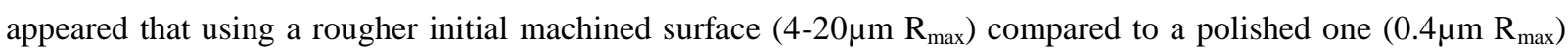
facilitated the formation of a compacted oxide layer. This was explained as being due to the fact that a rougher surface provided more recesses for the oxide to be compacted into.

More recently, Kato [19-21] investigated the addition of nano-scale particles in the size range 20-60nm. Concentrating on the room temperature sliding of $\mathrm{S} 45 \mathrm{C}$ surfaces with $\alpha-\mathrm{Fe}_{2} \mathrm{O}_{3}$ additions, he found that it was during the severe wear period that the compacted protective oxide layer began to form and that a critical load bearing area was necessary before the severe-mild wear transition could take place, after which further oxide 
formation was inhibited. There was a direct correlation to the severe-mild wear transition and the size of the nanoparticles which were added to the contact, with smaller particles reducing the length of the severe wear period.

Thus it appears that addition of nano scale oxide particles may play a role in reducing the severe running-in wear transition time and promote the formation of a wear protective oxide layer on metallic surfaces subject to sliding/fretting contacts at ambient and elevated temperatures. However, until now it appears that the addition of nano-particles to a tribological system to induce the severe-mild wear transition has only been as separate additions to the sliding contact area, which would be difficult to achieve in real engineering application. In the present work an attempt was made to make a homogeneous nano-particle coating of $\mathrm{Fe}$ on a ferrous substrate in order to facilitate the formation of a protective oxide layer at ambient and elevated temperatures. The aim was to reduce the severity of the running-in wear period and nucleate a protective oxide layer which would incorporate the tribo-products of the sliding process as an interfacial tribo-layer between the sliding metallic contact surfaces.

\section{Experimental}

Samples for flat-on-flat pin on plate wear testing were prepared from BS970 080M40 (EN8, AISI 1040 and S45C equivalents) low carbon mild steel, nominal composition shown in Table 1 and hardness $235 \pm 37 \mathrm{MPa}$. Plates $4 \mathrm{~mm}$ thick were cut to length $58 \mathrm{~mm} \times 13 \mathrm{~mm}$ and finished on the test surface with a ground finish perpendicular to the direction of sliding. Flat ended pin specimens (edge chamfered) were prepared to a diameter of $7 \mathrm{~mm}$ and thickness of $4 \mathrm{~mm}$ and ground to a 600 grit finish using $\mathrm{SiC}$ grinding paper. The respective average peak to valley heights $\left(R_{z}\right)$ of the plate and pin specimens were $1.30 \pm 0.29 \mu \mathrm{m}$ and $0.76 \pm 0.29 \mu \mathrm{m}$, measured using a Taylor Hobson diamond tip stylus with a profile length of $3 \mathrm{~mm}$ and a cut-off filter value of $0.8 \mathrm{~mm}$, corresponding to ISO 2CR standard.

\begin{tabular}{cccccc}
\multicolumn{7}{c}{ Table 1. Nominal composition (wt\%) of 080M40 [23]. } \\
\hline $\mathrm{C} \%$ & $\mathrm{Si} \%$ & $\mathrm{Mn} \%$ & $\mathrm{~S} \%$ & $\mathrm{P} \%$ & $\mathrm{Fe} \%$ \\
$0.35-0.45$ & $0.05-0.35$ & $0.60-1.00$ & $<0.06$ & $<0.06$ & Balance \\
\hline
\end{tabular}

Deposition of Fe nano-particles on both substrate surfaces was carried out by Mantis Deposition Ltd, Thame, UK, using a terminated gas condensation PVD process. The process utilises a high-pressure sputter source to generate Fe nano-particles from within a gas condensation zone in the deposition chamber. The gas flow within the condensation zone allows the nano-particles to be directed towards the EN8 substrate. Particle diameters in the range 5-10nm were selected using a mass quadrapole prior to deposition on the substrate surface, with the total coating thickness estimated at $600 \mathrm{~nm}$.

Tribological testing was carried out on flat-on-flat, self-aligning contact geometry, using a Phoenix Tribology TE77 high frequency reciprocating tribometer, represented by the schematic and sketch in Figure 1. Tests were carried out at a fix stroke length of $400 \mu \mathrm{m}, 31 \mathrm{~Hz}$ test frequency (corresponding to a sliding speed of $24.8 \mathrm{~mm} / \mathrm{s}$ ) and $40 \mathrm{~N}$ normal load, which corresponded to an apparent contact pressure of $1.04 \mathrm{MPa}$. The stroke length was deliberately keep short in order to confine the wear particles to the contact area, with the contact pressure chosen 
to be comparable to previous similar studies $[19,20]$. Tests were conducted at room temperature $\left(23-25^{\circ} \mathrm{C}, 28\right.$ $49 \%$ relative humidity), $300^{\circ} \mathrm{C}$ and $540^{\circ} \mathrm{C}$. Due to a program error during the room temperature coated test, a test time of 120 s instead of the 180 s (used for the higher temperature tests) was achieved, corresponding to sliding distances of $2.98 \mathrm{~m}$ and $4.46 \mathrm{~m}$, respectively. The remaining uncoated RT tests were continued at $120 \mathrm{~s}$ for comparison. The test lengths were based upon the time necessary for the establishment of a constant dynamic friction coefficient, i.e. the end of the 'running-in' period. For high temperature tests, the normal load was applied to the specimen, prior to heating to the desired test temperature at a rate of $10^{\circ} \mathrm{C}$ per minute. Once the test temperature had been reached, a dwell period of 30 minutes took place prior to the sliding test. A minimum of 3 repeat tests were carried out on uncoated specimens, however the limited availability of the experimental coatings made this impracticable for the coated samples. The contact potential was measured throughout each test over a $50 \mathrm{mV}$ range and was used to establish the formation of insulating oxide layers between the test surfaces. After testing, an Alicona Infinite Focus microscope was used to generate 3D surface reconstructions of the worn pin and plate surfaces, which allowed the respective volumes above and below a plane placed at the height of the original surface (determined from un-worn areas of the samples) to be calculated.
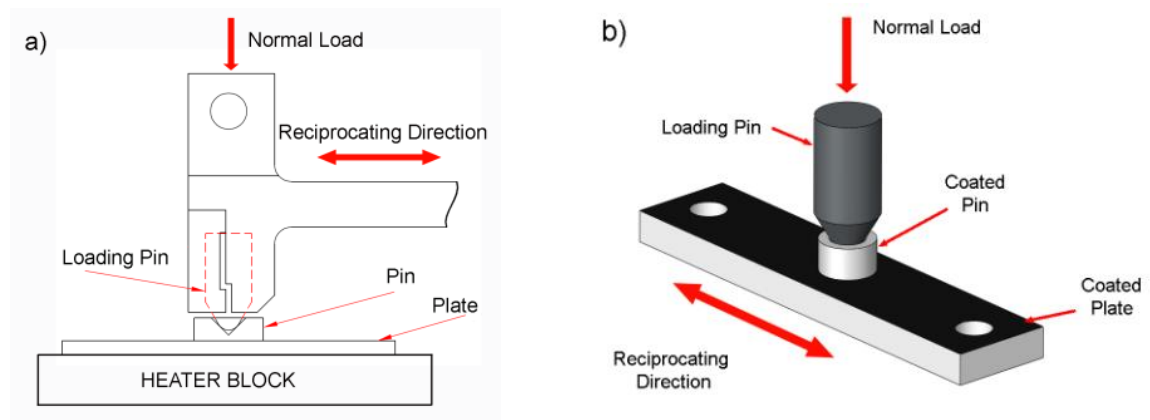

Figure 1. a) Schematical representation of the self-aligning pin on plate geometry in theTE-77 reciprocating tribometer and b) a sketch of the self-levelling contact geometry without the reciprocating arm in place.

A Zeiss NVision 40 dual-beam field emission gun (FEG) and focused ion beam (FIB) was using to examine and cross section areas of the contact surface from the coated pin specimens after sliding. In addition the FIB was used to make transmission electron microscopy lamella from selected areas of the worn surface, which were analysed using the STEM detector in the same machine. Finally, in-situ FIB secondary ion mass spectrometry (SIMS) depth profiling was carried out on the worn coated pin specimens using a Hiden Analytical quadrapole detector. A sputter area of $30 \times 30 \mu \mathrm{m}$ was used on each sample at a beam current of $13 \mathrm{nA}$ for 20 minutes. The relative depth of each depth profile was determined afterwards by measuring the depth of the sputter crater.

\section{Results}

\subsection{Deposited Coatings}

The as-received Fe nano-particle coated 080M40 surfaces were black in appearance. Figure 2 a) shows an SEM image of a coated surface. It was clear that the particles had followed the surface topography of the substrate 
material when deposited. A higher magnification image, Figure $2 \mathrm{~b}$ ), shows the individual nano-particles and how they appeared to coalesce into a "cauliflower pattern" of approximately $100 \mathrm{~nm}$ regions in size.
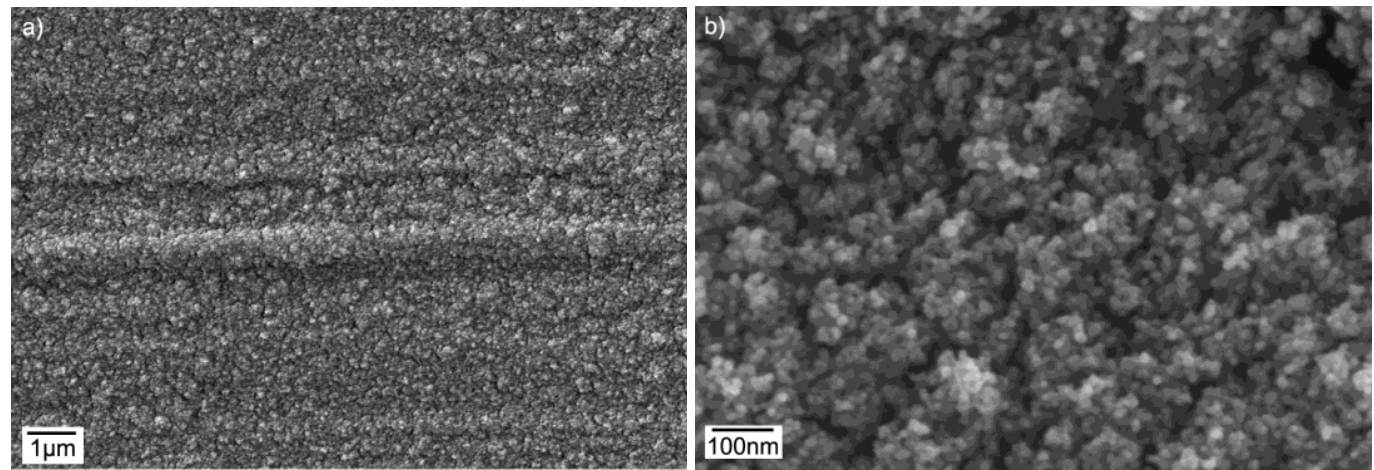

Figure 2. Secondary electron images of the as-deposited Fe nano-particle coated surface.

\subsection{Room Temperature Tests}

Figure 3 shows the friction coefficient recorded during sliding at room temperature. Both the uncoated and Fe nano-particle coated specimens exhibited a static friction coefficient of 0.2 . It would take approximately 10 seconds for the reciprocating motion to accelerate to the test frequency of $31 \mathrm{~Hz}$. During this time and thereafter, up to approximately 100 seconds, there was a steady increase in the coefficient of friction for all samples until a steady value had been obtained for the remainder of the test. The average dynamic friction coefficient at the end of the test for the uncoated sample surfaces was slightly lower than the coated surface, $0.60 \pm 0.02$ compared to 0.64 , with average friction coefficients over the entire test length $0.49 \pm 0.05$ and 0.52 , respectively. Contact potentials for both coated and uncoated tests remained almost zero throughout the duration of the tests $(<0.5 \mathrm{mV})$.
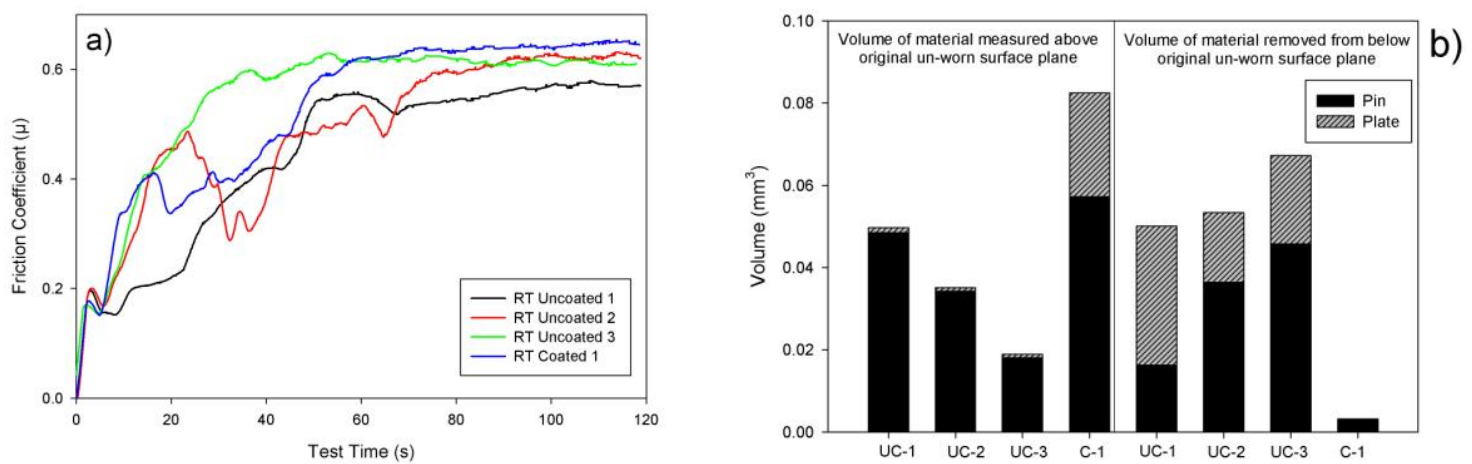

Figure 3. a) Friction coefficients vs time and b) 3-dimensional wear volume measurements for room temperature sliding tests of uncoated (UC) and coated (C) EN8 steel substrates.

The volume material measured as either loss (wear) or retention (tribo-material) from the original plane of the contact pin and plate surfaces, using 3D surface reconstructions, is shown in Figure $3 \mathrm{~b}$ ) and complimented by the 3D colour depth images shown in Figure 4 a)-d). From the wear volume data is can be seen that the volume of 
material removed from the uncoated surfaces at room temperature was a similar order of magnitude to that retained between the contact surfaces. It was also evident that most of the tribo-material retained in the contact for tests conducted on uncoated surfaces was adhered to the pin surface, rather than the plate. This can be seen in Figure 4 a) and b) where the 3D depth image shows that the pin exhibits areas that are both raised above and worn below the original worn surface, in contrast to the plate which only exhibits material removed from the wear surface. The coated surfaces, Figure 4 c) and d), show a minimal volume of material worn from below the original contact surface, but crucially, a higher volume of material retained within the contact area when compared to the uncoated surfaces. Surface material in this case was mainly removed from the contact pin, although both pin and plate retained tribo-material. Specific wear rates measured from the total volume of material removed from the worn surface were calculated to be $47.8 \times 10^{-5} \pm 7.6 \times 10^{-5} \mathrm{~mm}^{3} / \mathrm{Nm}$ for the uncoated surfaces compared to $2.7 \times$ $10^{-5} \mathrm{~mm}^{3} / \mathrm{Nm}$ for the experimental coating, an order of magnitude lower.
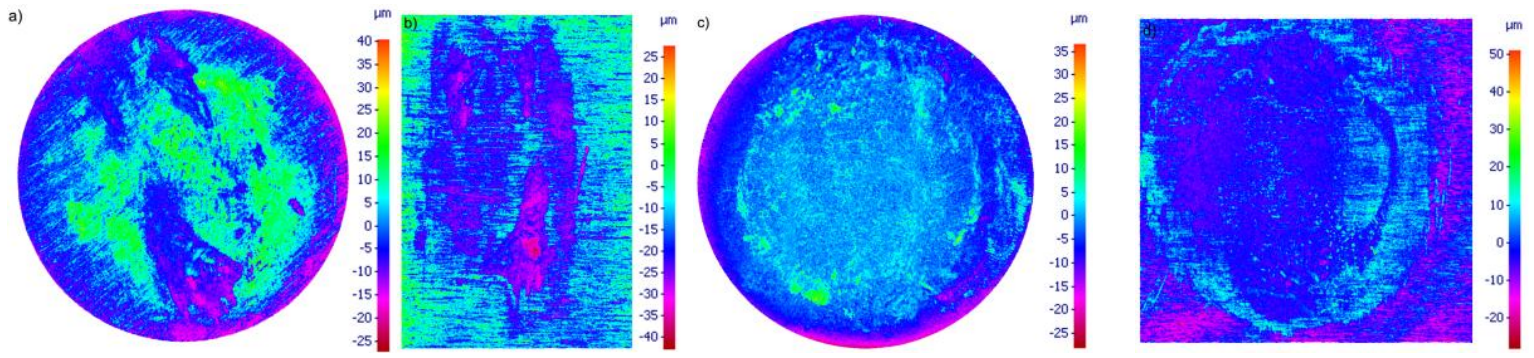

Figure 4. 3D surface reconstructions of the surfaces of the a) Uncoated 1 test pin and b) plate and c) Coated 1 test pin and d) plate, tested at room temperature. The pin diameter is $7 \mathrm{~mm}$.

SEM analysis of the raised volumes of tribo-material observed on the Coated 1 (C-1) pin surface after sliding for 120 s at room temperature revealed a number of interesting features, as shown in Figure 5 a)-d). The nanoparticle coating had been disrupted due to the sliding action, with areas of agglomerated particles observed at the leading edges of deformed substrate material, Figure 5 a). It was noted that such areas also had a propensity to retain larger wear debris fragments, Figure $5 \mathrm{~b}$ ), both from what appeared to be compacted regions of nanoparticles themselves, but also larger debris particles associated with worn substrate material. Another feature of interest was the appearance of porous structures adhered to the surface, Figure $5 \mathrm{c}$ ), which higher magnification showed were a mixture of particulate and wear debris material from the substrate, Figure $5 \mathrm{~d}$ ). 

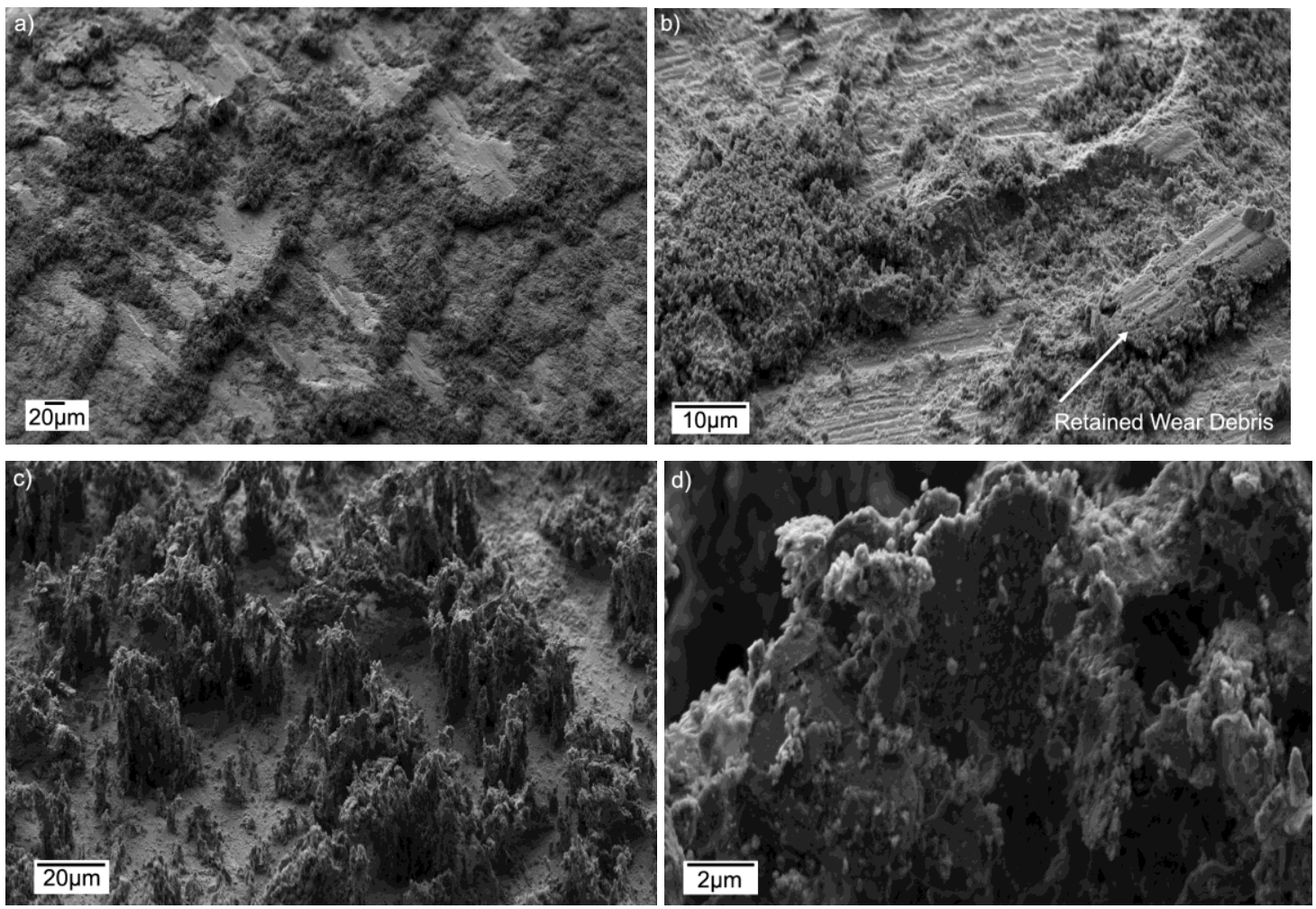

Figure 5. a) - d) Secondary electron images of the coated 080M40 pin surface after room temperature sliding for 120s; $55^{\circ}$ tilt away from surface normal.

\section{$3.3300^{\circ} \mathrm{C}$ Tests}

Figure 6 a) shows the friction coefficient and contact potential of tests conducted on coated and uncoated $080 \mathrm{M} 40$ substrates at $300^{\circ} \mathrm{C}$. The average dynamic friction coefficient of the uncoated $080 \mathrm{M} 40$ was slightly lower than those tested at room temperature, $0.40 \pm 0.01$ compared to $0.49 \pm 0.05$. The coated surface exhibited similar behaviour to the uncoated material, although a small reduction was observed after 115 seconds. Also of note was a double friction spike observed for all tests, where the friction coefficient rose to a maximum, dipped slightly, before rising to a second maximum and dropping to a constant value for the duration of the test. The position of the second peak correlated to the achievement of the test speed after 10 seconds of acceleration. Contact potential values for the uncoated 080M40 all exhibited an incubation time of between 80-150 seconds before increasing. In contrast, the Fe nano-particle coated surface showed a small increase much earlier, after approximately 30 seconds. However this returned to zero after 50 seconds and did not increase for the remainder of the test. 

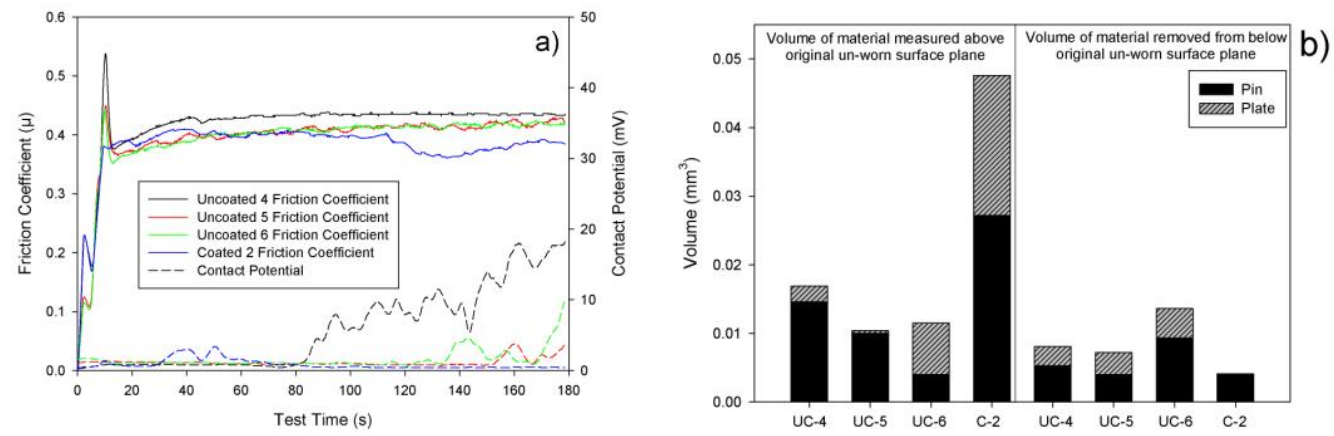

Figure 6. a) Friction coefficient and contact potential vs time and b) 3-dimensional wear volume measurements for tests conducted at $300^{\circ} \mathrm{C}$ on uncoated (UC) and coated (C) EN8 steel substrates.

Figure $6 \mathrm{~b}$ ) shows the wear volume calculated from 3-D surface reconstructions, examples of which are given in Figure 7. For the uncoated 080M40 surfaces, the volume of material removed from the contact surfaces was again similar to the amount observed on the worn surface. However, for the coated surface, it appeared that the Fe nano-particles assisted in retaining a higher volume of material in the tribo-contact region compared to the uncoated surfaces. The wear of the coated surface was confined to the plate only, as observed in Figure $7 \mathrm{c}$ ) and d), the pin exhibiting only volumes above the original contact surface. Whilst tribo-material was also observed above the original contact surface for the uncoated specimens, Figure 7 a), again like the uncoated room temperature samples, this was in addition to material removed as wear debris from surrounding regions. The uncoated plates, again, exhibited only material removal from the contact surface of the uncoated specimens. Two further observations were that the original grinding marks on the pre-test samples were still visible in the depth colour maps of the uncoated surfaces, Figure 7 a) and b), but less so on the coated surfaces after wear. Further, some areas of the worn coated surface appeared to have a concentric ring appearance to the volume of material which was above the original contact surface. Specific wear rates calculated from the amount of material removed from the wear surfaces were $5.4 \times 10^{-5} \pm 1.9 \times 10^{-5} \mathrm{~mm}^{3} / \mathrm{Nm}$ and $3.14 \times 10^{-5} \mathrm{~mm}^{3} / \mathrm{Nm}$ for the uncoated and coated specimens, respectively. For the uncoated surfaces, this value is significantly less compared to the tests conducted at room temperature, whereas for the coated surfaces the values were similar.
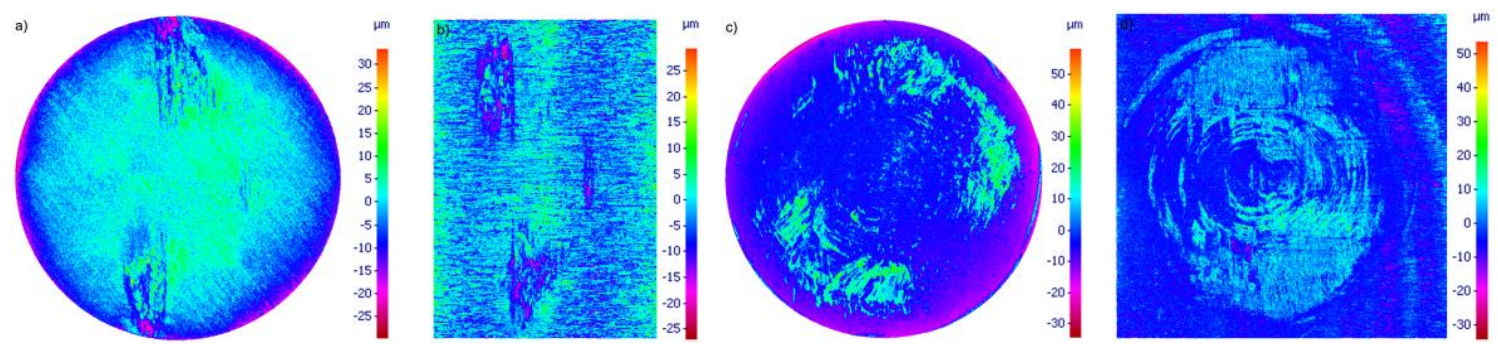

Figure 7. 3D surface reconstructions of the surfaces of the a) Uncoated 3 test pin and b) Plate and c) Coated 2 test pin and d) plate, tested at $300^{\circ} \mathrm{C}$. The pin diameter is $7 \mathrm{~mm}$. 
Scanning electron microscopy of the surface of the Fe nano-particle coated pin worn at $300^{\circ} \mathrm{C}$, Figure 8 a) and $b$ ), revealed that the raised volume of material on the pin surface observed in Figure 7 c) was wear debris which had been smeared into the original surface of the sample to form a tribo-layer. At higher magnification, Figure $8 \mathrm{~b}$ ), it was clear that the Fe-nano particle coating was still present on the unworn areas of the surface, with some of the particles appearing to coalesce into larger clumps.

The tribo-layer appeared to form on top of the nano-particulate layer, rather than in place of or below it and focused ion beam cross sections through this tribo-layer confirmed this was the case. Figure 9 a)-d) shows a number of STEM bright and dark field images of a cross section lamella prepared through the tribo-layer parallel to the sliding direction. Figure $9 \mathrm{~b}$ ) shows the un-deformed ferrite and pearlite grain structure at the base of the image [24] and how as the surface is approached, the refinement of the grains takes place to a sub-mircon level. The upper most surface in the image is a layer of carbon deposited during preparation of the lamella in order to protect the worn surface. Below the carbon layer appeared an intermittent white structure which was the Fe nanoparticulate layer. Further down was the deforming bulk microstructure. At higher magnification it became obvious that there was a tribo-layer at the upper surface which had formed on the nano-particle coating with what appeared considerable adhesion, i.e. no porosity and perhaps a columnar granular structure aligned perpendicular to both the sample surface and the sliding direction. Also observed was the coarsening of the nano-particles themselves, from an initial particle size observed in Figure 2 of 5-10nm to particles up to 100nm in size, although there still appeared to be a range of sizes up to this value. Closer inspection, Figure $9 \mathrm{~b}$ ), also suggested that there was a well adhered layer of Fe nano-particles on the deformed 080M40 substrate, above which existed the coarser and slightly more porous nano-particle layer, finally followed by the surface contact tribo-layer.
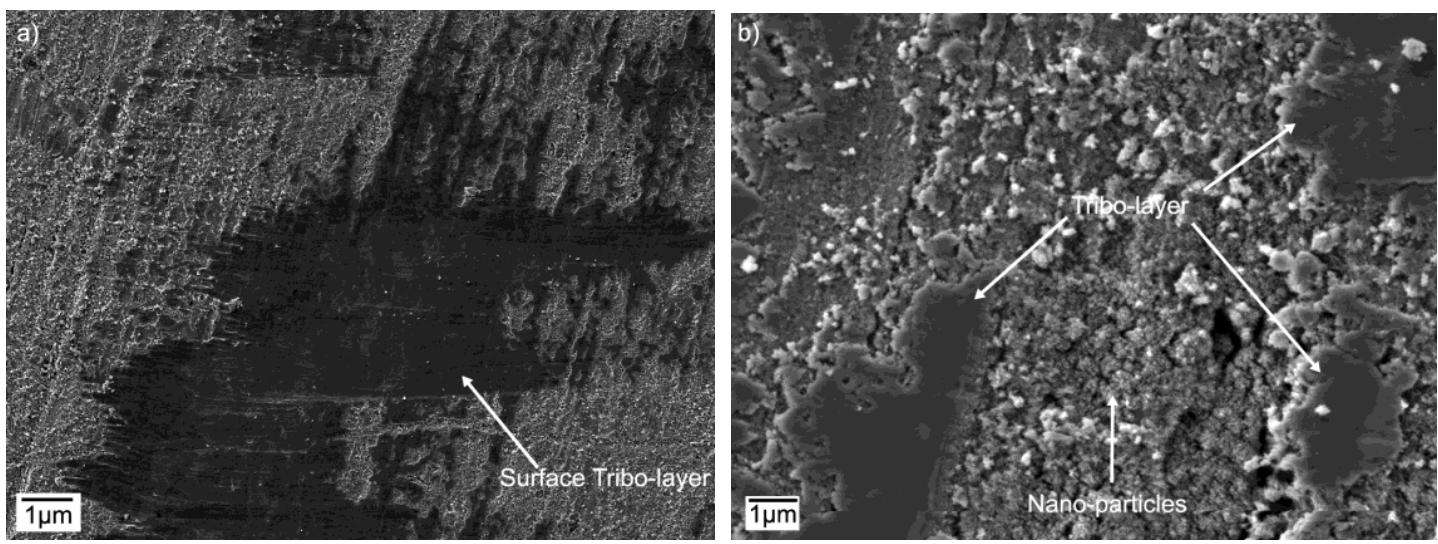

Figure 8. Secondary electron images of the Coated $2(\mathrm{C}-2)$ surface after testing at $300^{\circ} \mathrm{C}$. 

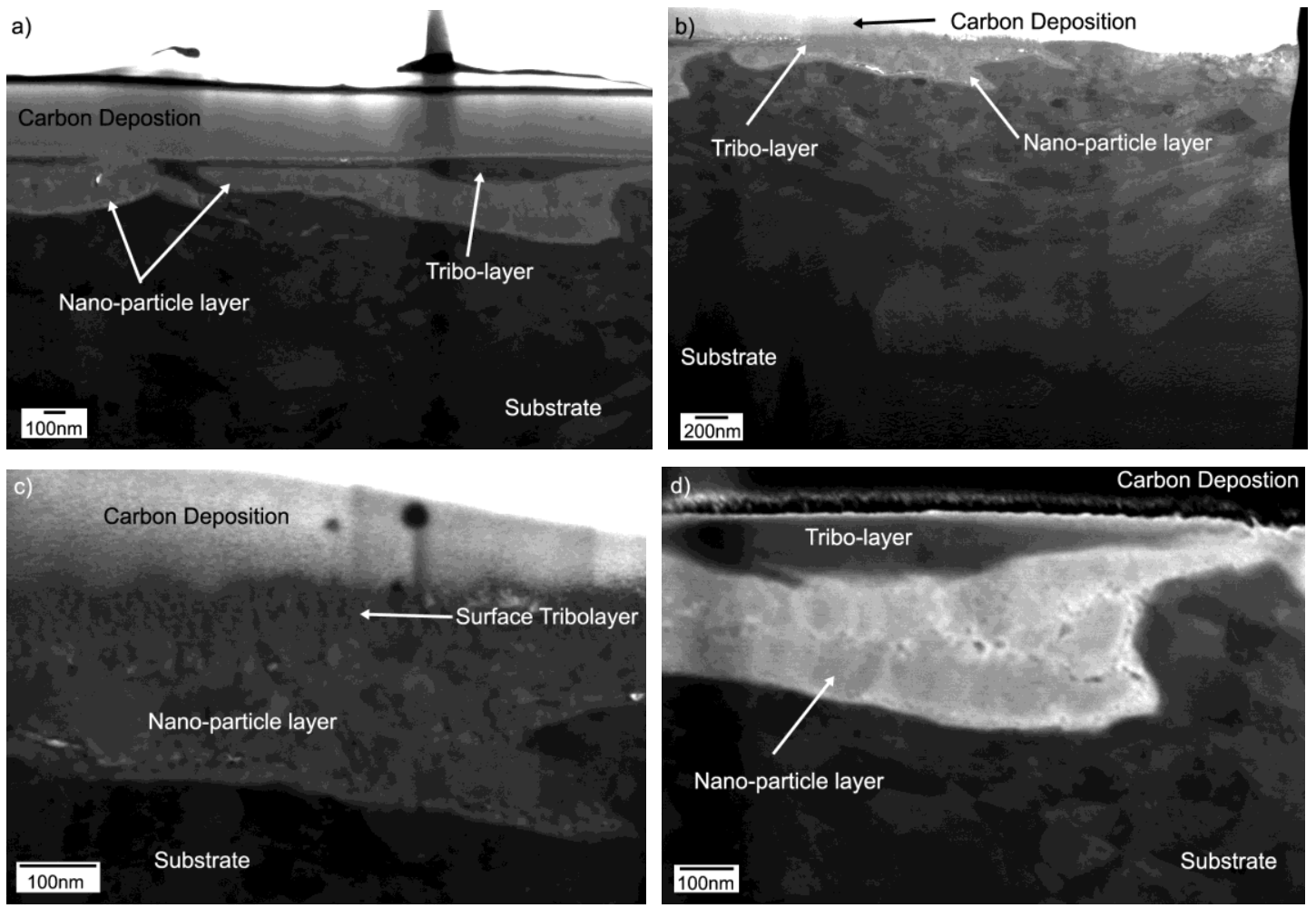

Figure 9. STEM bright and dark field images of a) - b) a FIB cross section through tribo-layer from the Coated 2 pin; c) and d) higher magnification of the contact surface region.

\section{$3.4540^{\circ} \mathrm{C}$ Tests}

Figure 10 a) shows friction coefficient and contact potential of tests conducted on coated and uncoated $080 \mathrm{M} 40$ at $540^{\circ} \mathrm{C}$. Interestingly, friction coefficients of the uncoated samples were similar to tests carried out at $300^{\circ} \mathrm{C}, 0.39 \pm 0.01$. The Fe nano-particle coated surface, exhibited a similar initial dynamic friction coefficient to the uncoated material and a reduction as the test progressed, similar to the coated surface tested at $300^{\circ} \mathrm{C}$. In contrast to the tests conducted at $300^{\circ} \mathrm{C}$, no incubation period was observed for the increase in contact potential measurements, values increasing from $3 \mathrm{mV}$ to $4-9 \mathrm{mV}$ moments after the full test speed had been reached. This initial increase was followed by steady values for the remainder of the test, the coated sample exhibiting the highest contact potential compared to the uncoated specimens, suggesting a greater proportion of non-conducting oxide present on the surface. 

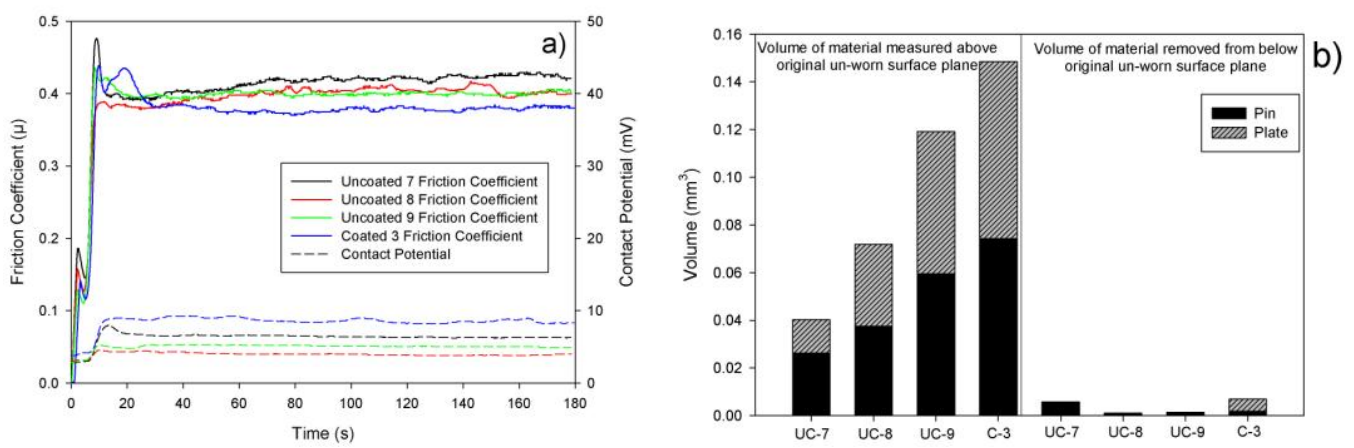

Figure 10. a) Friction coefficient and contact potential and b) Wear volumes for tests conducted at $540^{\circ} \mathrm{C}$ on uncoated (UC) and coated (C) EN8 steel substrates.

Measurements of wear volumes, Figure 10 b) from 3-dimensional surface reconstructions, Figure 11 a) - d) indicated that the nano-particle coated surface once again exhibited the highest volume of retained surface material above the plane of the original worn surface, although there was considerable variation in the uncoated results. It was noted that the relative proportions of tribo-material above the original worn surfaces were approximately equal for both the pin and the plate for all tests. Significantly, the volume measured to be retained on the worn surfaces was much higher than that measured to have been removed from both uncoated and coated tests. In contrast to previous tests, the results in Figure $10 \mathrm{~b}$ ) indicated that the coated surface did not exhibit any advantage over the uncoated surface in terms of wear volume removed. Specific wear rates for the uncoated and coated surfaces were $1.51 \times 10^{-5} \pm 1.46 \times 10^{-5} \mathrm{~mm}^{3} / \mathrm{Nm}$ and $6.55 \times 10^{-5} \mathrm{~mm}^{3} / \mathrm{Nm}$, respectively, the high standard deviation error of the former reflecting the scatter in results. Such values were similar to those observed for coated specimens at room temperature and $300^{\circ} \mathrm{C}$, although in this case the coated sample appeared to perform worse compared to the uncoated specimens.
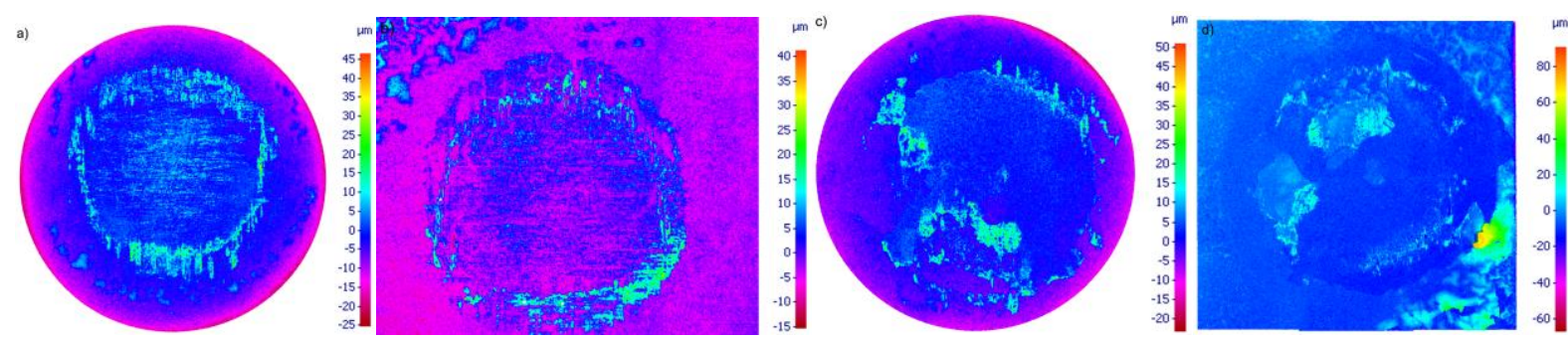

Figure 11. 3D surface reconstructions of the surfaces of the a) Uncoated 9 pin and b) plate and c) Coated 3 test pin and d) plate after testing at $540^{\circ} \mathrm{C}$. The pin diameter is $7 \mathrm{~mm}$.

Scanning electron microscopy of the worn Coated 3 pin surface confirmed the presence of a raised plateau above the plane of the original coated surface, Figure 12 a). This was similar to that seen for the coated samples at $300^{\circ} \mathrm{C}$, although covering a much great proportion of the pin and in areas had spalled to reveal the original coated surface. FIB cross sections through the tribo-layer indicated a layer thickness of approximately $2 \mu \mathrm{m}$, although this 
would vary with the depth of the wear tracks observed (i.e. a function of the surface roughness). The layer was composed of nano-crystallite grains but was poorly attached to the 080M40 surface. The substrate microstructure appeared deformed towards the direction of sliding, with elongated micron and sub-micron grains and a total depth of deformation $(>4 \mu \mathrm{m})$ greater than that observed at $300^{\circ} \mathrm{C}$. A TEM lamella from the tribo-layer, Figure 13 a) and b) revealed a compact surface layer, with an increase in porosity further away from the contact surface. Interestingly, the microstructure of the tribo-layer exhibited a grain refinement as the contact surface was approached such that the upper surface was itself deformed towards the sliding direction and exhibited nanograins of the order of $20-100 \mathrm{~nm}$. The extreme upper surface of the contact showed a layer of material approximately $20 \mathrm{~nm}$ thick and it was believed that this layer may have formed after the sliding test had ceased and the sample cooled to ambient temperature from $540^{\circ} \mathrm{C}$. The appearance of what appeared to be $10 \mathrm{~nm}$ surface bumps were believed to be an artefact formed during the deposition of the protective carbon film, when the $\mathrm{Ga}^{+}$ ion beam sometimes interacts and sputters material from the upper $20 \mathrm{~nm}$ surface before inducing chemical deposition of the carbon layer.
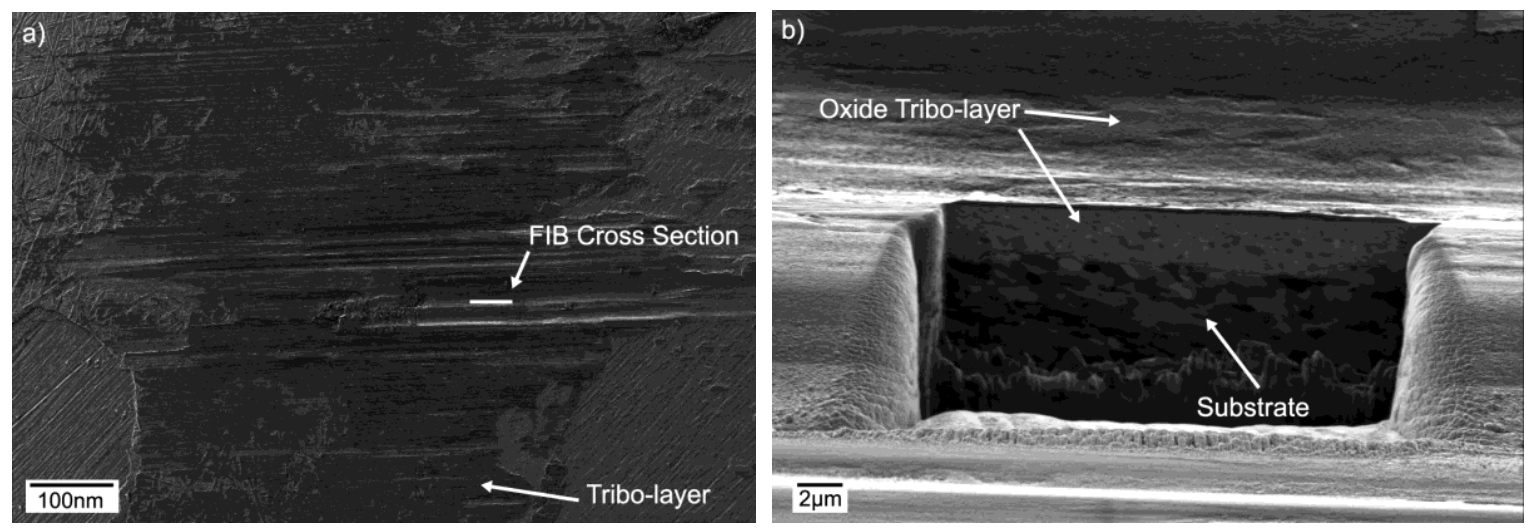

Figure 12. a) Secondary electron image of the worn Coated 3 surface after testing at $540^{\circ} \mathrm{C}$ and b) FIB cross section through the upper tribo-layer indicated in a).
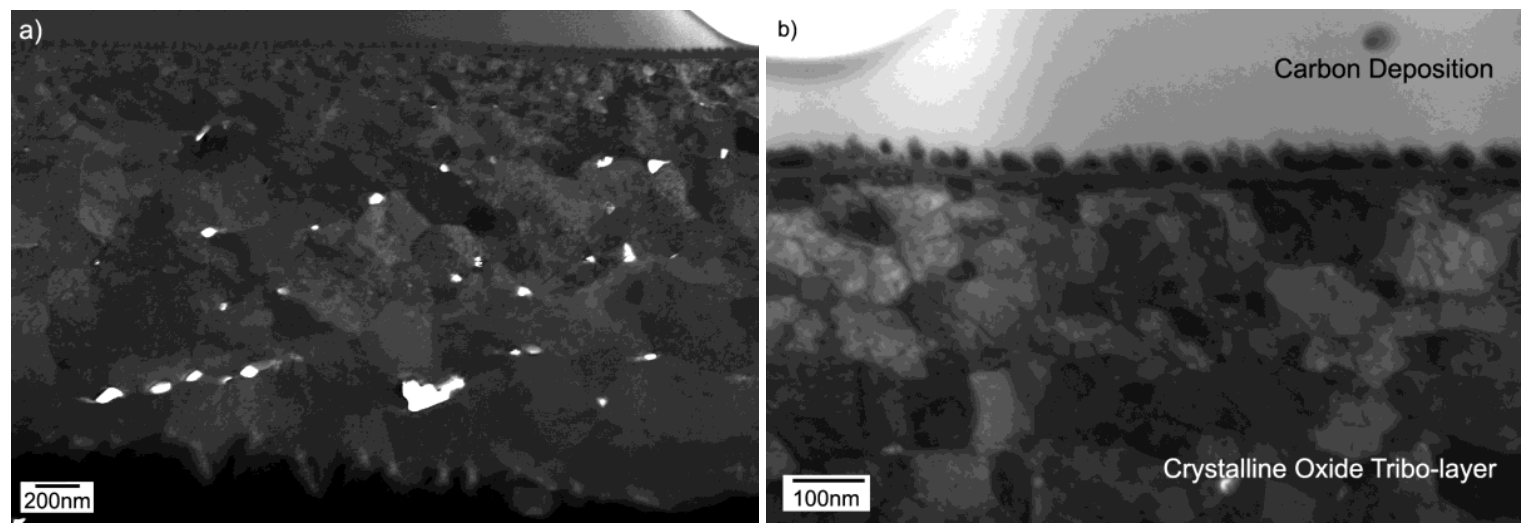

Figure 13. Bright and dark field STEM images from within the tribo-layer. 


\section{Secondary Ion Mass Spectrometry}

SIMS depth profiling was carried out on the tribo-layers of the coated samples in order to determine the composition of the surface layer in comparison to the uncoated 080M40 substrate, Figure 14 a) - c). Samples were handled with protective gloves before and after testing and stored in individual cases to avoid contamination of surface species. It was surprising to find a number of contaminant elements within the steel substrate itself, including $\mathrm{Al}$ and As. A strong $\mathrm{O}^{16-}$ signal was present for all surfaces investigated, whilst positive ion species which were prominent during an atomic mass spectra (0-200 amu) from the surface included $\mathrm{Al}^{+}$and $\mathrm{P}^{+}$as well as $\mathrm{AlO}^{+}, \mathrm{PO}^{+}$and $\mathrm{FeOH}^{+}$. Whilst the profiles were collected for the same time length $(20 \mathrm{mins})$, because of the difference in sputter rates of the surface oxide species for the room temperature compared to the $300^{\circ} \mathrm{C}$ and $540^{\circ} \mathrm{C}$ tests, the total depth represented by the room temperature profile was $12 \mu \mathrm{m}$, compared to $5 \mu \mathrm{m}$ for the elevated temperature tests.
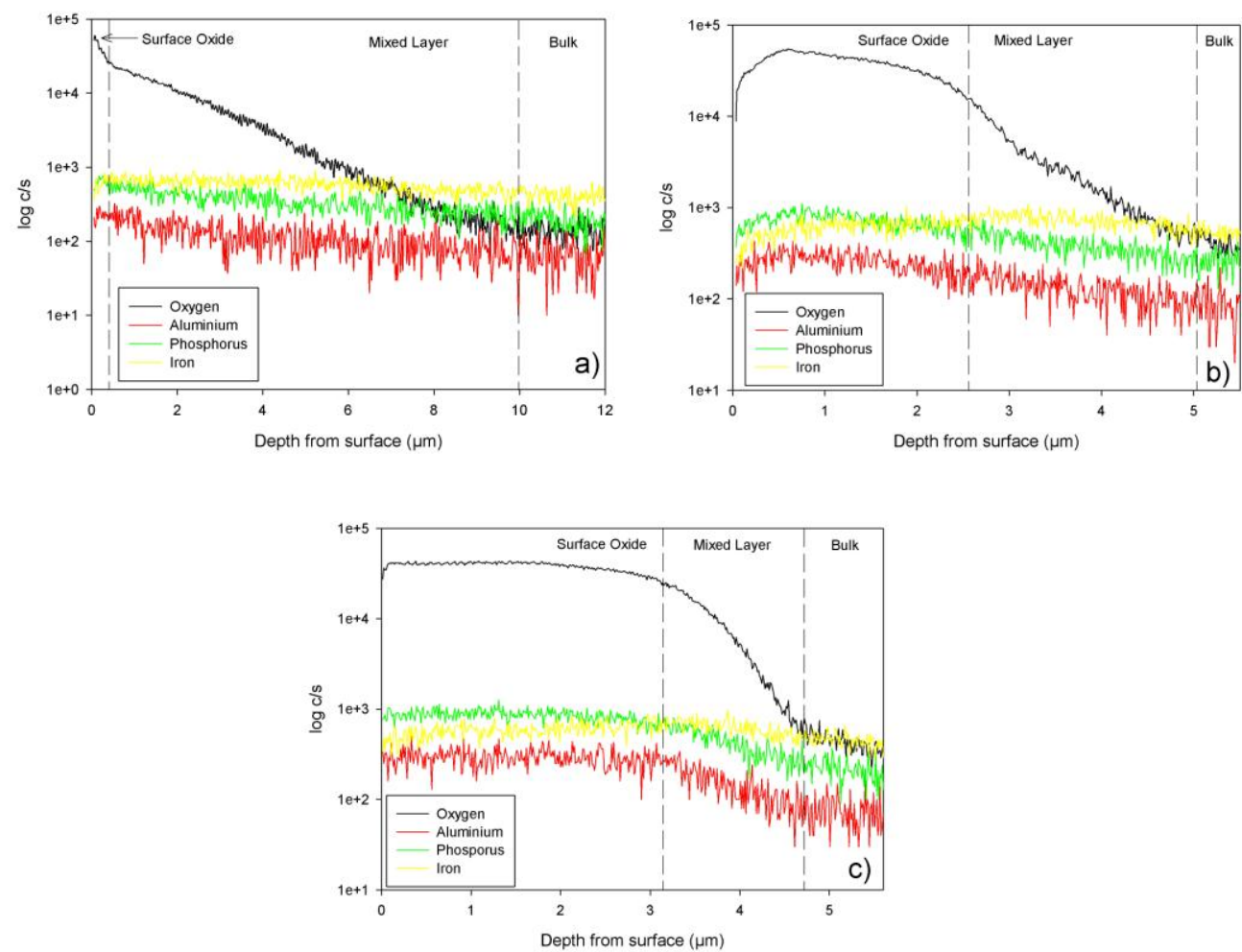

Figure 14. SIMS depth profiles from tribo-layers formed on the coated pin specimens at a) room temperature, b) $300^{\circ} \mathrm{C}$ and c) $540^{\circ} \mathrm{C}$.

The depth profile through the accumulated material observed on the coated sample tested at room temperature, similar to that seen in Figure 5 a), showed that only the extreme upper surface had oxidised with a composition different to the bulk and that the majority of the tribo-layer was composed of a mixed layer which incorporated progressively less oxide as the bulk substrate was approached. The level of Al and P were slightly 
higher at the contact surface, decreasing slightly to the bulk substrate composition within the mixed tribo-layer region.

SIMS depth profiling of the $300^{\circ} \mathrm{C}$ sample showed an interesting correlation in the trend between the oxygen and aluminium and phosphorus signals, both increasing to a maximum level just below the worn surface, before dropping off within the mixed layer region to bulk substrate values, similar to the room temperature depth profile behaviour at the substrate interface. The relative proportion of iron in the surface oxide layer was notable lower than in the mixed region, itself slightly higher than the bulk concentration.

Finally, the analysis conducted on the tribo-layer generated on the coated surface tested at $540^{\circ} \mathrm{C}$, Figure 14 c), showed the presence of an oxide film with a mixed composition, the presence of aluminium and phosphorus being notably higher. A similar trend to the $300^{\circ} \mathrm{C}$ analysis was observed with the concentration of iron being higher next to the substrate interface, before decreasing to bulk composition values. It was interesting to note that both the surface oxide had increased in thickness with increasing temperature, as could be inferred from the electron micrographs, but also that the thickness of the mixed region of decreasing oxide concentration decreased with an increase in test temperature.

\section{Discussion}

\subsection{Deposited Coatings}

The Fe nano-particle coatings were evenly distributed over the ground 080M40 substrate surfaces, following the surface topography rather than filling in surface recesses, as is typical in line-of-sight PVD deposition processes. The black appearance of the coating would suggest that the upper layer had oxidised, perhaps to $\mathrm{Fe}_{2} \mathrm{O}_{3}$, upon removal of the coating to the atmosphere from the PVD coating chamber. The particles appeared to agglomerate slightly into clumps approximately $100 \mathrm{~nm}$ in size and it was believed that this was the reason such a size distribution was observed in the cross sectional STEM images taken from the coated pin after sliding at $300^{\circ} \mathrm{C}$, Figure $\left.9 \mathrm{c}\right)$ and d).

\subsection{Room Temperature Tests}

The static friction coefficient of tests at ambient conditions was similar, just below 0.2. The increase in dynamic friction coefficient to steady values after 100s represented the running-in period of wear which contributed to the initial formation of interfacial tribo-layers observed in the 3D surface profiles [1,2]. There was no evidence from the friction or contact potential data for the test length investigated that the Fe nano-particle coated surface accelerated this formation, as had been reported in other studies, where the artificial introduction of oxide particles reduced the incubation time for a transition from severe to mild wear $[6,17,18,20]$. Thus severe wear still was considered to be the dominant wear mechanism after 120 seconds sliding, consistent with the appraisal of Quinn's surfaces when sliding the same alloy at room temperature [3]. During Kato's work [19,20] the transition from severe to mild wear took place after 30-40m sliding distance, although their methodology did 
use a pin-on-disc geometry which would have required multiple passes to form a proportionally load bearing oxide film. It was interesting to note in that work and also shown here, that the oxide film formation appeared preferentially on the pin surface over the plate, at least for the uncoated surfaces. This was attributed to the pin surface being in continuous sliding contact, whilst the plate surface would only have experienced intermittent contact. The results from the 3D surface depth profiles in this work did however indicate that the coated surfaces had a higher propensity to retain wear debris particles and that the damage to the underlying substrate was minimal compared to the uncoated surfaces. It would be interesting to test such coatings for a longer length scale at room temperature to determine the tribo-film response and wear rates over extended periods of sliding contact.

Analysis of the raised tribo-layer showed that it was composed of a thin layer of oxidised nano-particles. The action of asperity contact had caused them to become agglomerated, compacted and able to trap wear debris within the contact geometry, thus allowing the formation of an intermediate tribo-layer between both pin and plate surfaces. Tribo-oxidation would then progress only at the real areas of contact [1] through asperity flash temperature heating. The black appearance of the oxide scale would infer an $\mathrm{Fe}_{2} \mathrm{O}_{3}$ type structure. The thickness of the surface oxide layer was only of the order 500nm (estimated from SIMS analysis) and thus the oxidation and subsequent formation of the interfacial tribo-film was dependent solely on the asperity flash temperature rise, a further reason the transition point (from contact potential measurements) was not observed. This work is therefore in good agreement with numerous studies which show that when wear debris is retained with the contact, wear rates are generally lower $[9,10,12-15,17,22,25]$ whilst conversely for contacts where wear debris is removed from the system, (either by a geometric configuration such that gravity ensures wear debris particles drop out or by mechanical removal with a brush or high pressure jet of air) wear rates increase significantly [22,26].

The original grinding marks, visible on the uncoated surfaces, were not easily discernable on the worn coated surfaces (except on the plate surface, outside of the contact area - Figure $4 \mathrm{~d}$ )) with very little evidence of wear below the contact surface. Thus the Fe nano-particles assisted in the distribution of the contact load across the apparent area of contact, filled in the valleys of the ground surface (as observed by Iwabuchi $[17,18]$ ) in a similar manner to that observed for modern lubricants, where metallic nano-particle additions perform a similar role as 'pseudo-boundary lubricants' [27-29] . Material was still removed from the contact surface during the running in period (hence a similar friction coefficient to the uncoated surfaces) but this tended to be more a removal of the surface roughness peaks rather than the severe wear of material observed in the uncoated tests. The higher proportion of retained debris observed in the electron micrographs would then go on to form a protective oxide layer through oxidation by asperity flash temperature rises. This is one of the main differences between room and elevated temperature oxidation rates, as only the surface of the contact tribo-film was oxygen rich, the SIMS data suggesting a larger mixed debris layer existing underneath, next to the substrate.

\section{$5.3300^{\circ} \mathrm{C}$ Tests}

The lower average dynamic friction coefficient observed at both elevated temperature tests was considered to be a direct result of a more comprehensive oxide film formation and was consistent with similar elevated 
temperature tribology studies on this alloy [3]. The substrate material may also have been an influence as 080M40 is not noted for its high temperature properties and thus a 30 minute dwell at elevated temperatures may have tempered the microstructure [24].

The formation of a protective oxide film, signalling the transition from a severe to mild wear regime, was initially observed at $300^{\circ} \mathrm{C}$ for the coated samples, suggesting again that the nano particles aided the nucleation of an oxide tribo-layer faster than an uncoated surface, which has to go through the mechanism of generating and deforming wear debris from the substrate to the nano-scale $[10,12,14,25]$. For the uncoated sample this resulted in material loss from the contact area until a sufficient amount of wear debris had nucleated the formation of a comprehensive oxide film. Despite the coated surface initiating a more rapid formation of an oxide layer, the contact potential measurements showed this was not sustained, unlike the uncoated surfaces which all showed a steady increase towards the end of the test. This can be explained by the apparent increase in the real area of contact, observed in Figure 7 d), which would have resulted in a lower contact potential because of the increase in number of asperity contacts, but which would simultaneously allowed the ongoing formation of the intermediate oxide tribo-layer.

The SIMS depth profiling of the tribo-layer at this temperature further provided evidence of the surface oxide layer formation, confirming the composition to be a mixture of elements from the bulk material, a classic example of protective oxide film formation [11]. The reason that the peak of the oxygen signal was observed below the worn surface was attributed to the pin and plate samples being separated from within an oxide film layer, rather than at the contact surface itself, where the peak would be expected due to higher asperity flash temperatures. Interestingly, the concentration of Fe was observed to increase within the mixed layer region before again decreasing to bulk values, a reflection of the presence of the Fe nano-particle interfacial layer between the surface oxide species and the substrate material, as observed in the STEM images.

The volume of material removed from the uncoated sample surfaces was similar to that retained as a surface tribo-film, whereas the coated surface had a much smaller volume removed from the substrate, but a higher retention of material as a tribo-film. This can be explained by two factors: when metallic debris undergoes oxidation there is an associated volume expansion due to the lower density of iron oxide species compared to iron, but also because, as shown in this and other works [30-32], the surface oxide film generated as a result of tribooxidation is a somewhat porous structure. The material in the tribo-contact was not all metallic debris of the same density as the substrate but tribo-oxidation products, as proved by the contact potential and SIMS data. Taking into account this volume expansion, material from the uncoated surfaces must have been lost from the contact area, else the volume retained would be similar to that seen for the coated sample. The low wear rates of the coated specimens again reflect that material worn from the substrate was retained and re-worked, possibly into wear scars, such that the surface of the coated samples appeared with minimal wear volume losses. The confinement of the majority of wear in the uncoated samples to discreet areas near the edge of the pin was further evidence of a more severe wear period, in contrast to the much smoother coated samples, Figure 7 c) and d), which exhibited evidence of a much larger real contact area that would have reduced the local contact stresses. In this case as at room temperature, the Fe nano-particles facilitated the smoothing of the surface roughness of the 
initial contact surfaces, but crucially allowed the wear debris and tribo-layer to bond to the contact surface and remain within the tribo-contact, again effectively protecting it from severe wear, as observed in the STEM images of Figure 9.

\section{$5.4540^{\circ} \mathrm{C}$ Tests}

Tests conducted at $540^{\circ} \mathrm{C}$ were very similar is their friction behaviour compared to tests at $300^{\circ} \mathrm{C}$, for the same reasons as outlined previously, with the coated sample again exhibiting a slightly lower friction coefficient. Wear of the substrate material was minimal as the surface had pre-oxidised during heating (contact potential measurements of $3 \mathrm{mV}$ compared to $0.3-1.6 \mathrm{mV}$ for $300^{\circ} \mathrm{C}$ test and $0.5 \mathrm{mV}$ for room temperature tests) and the transition from severe to mild wear was almost immediate upon sliding, confirmed by constant contact potential measurements established a couple of seconds after the test had attained the running speed. Such behaviour was in agreement with Quinn's experiments at $500^{\circ} \mathrm{C}$ with EN8 material in like on like contact, exhibiting only a mild oxidational wear behaviour [3]. The volume of material measured above the worn surface was again greatest for the coated sample surface, although, despite showing a large scatter in results from the uncoated surfaces, the difference was much smaller compared to tests carried out at lower temperatures. In this case, the oxidation of all test surfaces prior to sliding was responsible for the large volume of material retained above the original contact surface, shielding the substrate from severe wear by adhesion or ploughing.

As expected, the oxide tribo-layer was much thicker compared to tests at lower temperatures with evidence of its load bearing capacity shown in Figure 13, where grain sizes progressively decreased as the surface was approached from the bulk, and aspect ratios increased towards the asperity sliding direction [33]. The formation of a compact oxide tribo-layer on top of a more porous debris structure demonstrates classic 'glaze' layer formation, although the term may not be universally applied to the present work as such layers are often associated with a significant reduction in friction [30-33]. An increased depth of deformation of the substrate was also noted, with the absence of large pearlite and ferrite grains observed at $300^{\circ} \mathrm{C}$, rather a surface deformed layer aligned towards the individual asperity sliding direction, again as a result of tempering due to the elevated temperature. Detachment of the oxide film from the substrate in accordance with the theory of oxidational wear was also observed [1], where detachment of oxide plateaux occurs at a critical thickness, although it was hard to determine whether this was also due to the contact surfaces being separated after cooling due to different thermal expansion coefficients.

SIMS depth profile results for the $540^{\circ} \mathrm{C}$ test correlated well with the SEM results in terms of the thickness of the oxide film being greatest for the highest temperature test, as expected. The chemical composition indicated that the film was richer in aluminium and phosphorus compared to tests at lower temperature, indicating that bulk diffusion of elements from the substrate occurred. A thinner mixed layer thickness was also indicative of the diffusion of oxygen in the opposite direction, resulting in a more complete reaction with the tribo-layer species. Although stoichiometry could not be determined by the methods described here, for elevated temperature tests the formation of $\mathrm{Fe}_{3} \mathrm{O}_{4}$ was inferred from the reddish nature of the test surfaces, although this may have been a mix of 
$\mathrm{Fe}_{2} \mathrm{O}_{3}$ and $\mathrm{FeO}$ in the $540^{\circ} \mathrm{C}$ test as the latter forms just above, at $570^{\circ} \mathrm{C}$. Further work is required to correlate the SIMS results to the crystallographic structure of the surface oxide.

At $540^{\circ} \mathrm{C}$ there was little evidence of the presence of the original Fe nano-particles, although given the high surface area of such small dimensions, it would be expected that oxidation had progressed rapidly at this temperature. However, the concentration of Fe was slightly higher at the substrate/tribo-layer interface, therefore it is postulated that the particles may have assisted in the adhesion of the static oxide layer which formed during the test dwell period at $540^{\circ} \mathrm{C}$ and this then contributed to the higher volume of oxide tribo-layer observed on the worn surface for the coated specimen, although static scratch tests would have to be carried out to determine this hypothesis in future work.

\section{Summary and Conclusions}

Room and high temperature testing of Fe nano-particle coatings showed that they could potentially be used for countering severe wear during the running-in period of initial sliding of metallic components, perhaps in the aerospace, automotive and energy sectors. Indeed, iron oxide spray coatings are already utilised as wear resistant coatings for cylinder liners in the automotive industry [34]. The incorporation of a sacrificial metal nano-particle coated layer which promotes fast establishment of a mild wear regime upon running-in, perhaps complimented with advanced nano-particle additive containing lubricants [27-29], could have an important contribution to make to the control of tribology during the initial and most critical period of component running-in sliding wear. The main conclusions of this work are summarised below:

- Fe nano-particles were successfully deposited to a thickness of $600 \mathrm{~nm}$ using a terminated gas condensation PVD process on an 080M40 substrate and used as tribological coatings at room and elevated temperatures.

- The coatings increased the propensity for the formation of a protective oxide tribo-layer composed of Fe nano-particles and material removed from the steel substrate, at ambient and elevated temperatures, above the plane of the original contacting surfaces. Fe nano-particle coatings provided a seed layer upon which protective oxide tribo-layers could form on metallic substrates, filling surface recesses and promoting a transition to a mild wear regime.

- The protective oxide tribo-layer increased in thickness with temperature and had a mixed composition of elements from the bulk substrate material.

- Although coated surfaces promoted a transition from a severe to mild oxidational wear mechanism, temperatures ambient and flash temperature in the contact appeared too low to promote sintering and therefore formation of a low friction oxide 'glaze' layer.

- This work has shown the feasibility of using nano-particle deposited coatings to improve the running in wear behaviour of metallic engineering substrates and promote the transition from severe to mild wear without significant damage to the contacting surface. Further work is required in order to optimise the 
coating chemistry to achieve a favourable 'glaze' structure and also to improve coating adhesion for robust engineering applications.

\section{Acknowledgements}

The authors would like to acknowledge access to the Southampton Nanofabrication Centre (SNC) for use of the Zeiss NVision 40 dual beam FIB-SEM and funding for the project from EPSRC Science and Innovation fund $\mathrm{EP} / \mathrm{F} 034296 / 1$.

\section{References}

[1] T.F.J. Quinn, Review of oxidational wear:: Part I: The origins of oxidational wear, Tribology International 16 (1983) 257-271

[2] T.F.J. Quinn, Oxidational wear modeling .1, Wear 153 (1992) 179-200

[3] T.F.J. Quinn, The oxidational wear of low alloy steels, Tribology International 35 (2002) 691-715

[4] W. Hirst, J.K. Lancaster, The influence of oxide and lubricant films on the friction and surface damage of metals, Proceedings of the Royal Society of London. Series A, Mathematical and Physical Sciences 223 (1954) 324-338

[5] W. Hirst, J.K. Lancaster, Surface film formation and metallic wear, Journal of Applied Physics 27 (1956) 1057-1065

[6] J. Lancaster, The formation of surface films at the transition between mild and severe metallic wear, Proceedings of the Royal Society of London. Series A, Mathematical and Physical Sciences (1963) 466-483

[7] E. Rabinowicz, Lubrication of metal surfaces by oxide films, ASLE TRANS 10 (1967) 400-407

[8] H.L. Du, P.K. Datta, I. Inman, R. Geurts, C. Kubel, Microscopy of wear affected surface produced during sliding of Nimonic 80A against Stellite 6 at 20 degrees C, Materials Science and Engineering a-Structural Materials Properties Microstructure and Processing 357 (2003) 412-422

[9] I.A. Inman, P.K. Datta, H.L. Du, J.S. Bumell-Gray, S. Pieugalski, Q. Luo, Studies of high temperature sliding wear of metallic dissimilar interfaces, Tribology International 38 (2005) 812-823

[10] F.H. Stott, J. Glascott, G.C. Wood, Factors affecting the progressive development of wear-protective oxides on iron-base alloys during sliding at elevated-temperatures, Wear 97 (1984) 93-106

[11] F.H. Stott, J. Glascott, G.C. Wood, The sliding wear of commercial Fe-12-percent Cr alloys at hightemperature, Wear 101 (1985) 311-324

[12] F.H. Stott, D.S. Lin, G.C. Wood, Structure and mechanism of formation of glaze oxide layers produced on nickel-based alloys during wear at high-temperatures, Corrosion Science 13 (1973) 449-\&

[13] F.H. Stott, G.C. Wood, Influence of oxides on friction and wear of alloys, Tribology International 11 (1978) 211-218 
[14] J.R. Jiang, F.H. Stott, M.M. Stack, The role of triboparticulates in dry sliding wear, Tribology International 31 (1998) 245-256

[15] F. Stott, High-temperature sliding wear of metals, Tribology International 35 (2002) 489-495

[16] J.R. Jiang, F.H. Stott, M.M. Stack, A generic model for dry sliding wear of metals at elevated temperatures, Wear 256 (2004) 973-985

[17] A. Iwabuchi, The role of oxide particles in the fretting wear of mild-steel, Wear 151 (1991) 301-311

[18] A. Iwabuchi, H. Kubosawa, K. Hori, The dependence of the transition from severe to mild wear on load and surface-roughness when the oxide particles are supplied before sliding, Wear 139 (1990) 319-333

[19] H. Kato, Severe-mild wear transition by supply of oxide particles on sliding surface, Wear 255 (2003) 426429

[20] H. Kato, Effects of supply of fine oxide particles onto rubbing steel surfaces on severe-mild wear transition and oxide film formation, Tribology International 41 (2008) 735-742

[21] H. Kato, K. Komai, Tribofilm formation and mild wear by tribo-sintering of nanometer-sized oxide particles on rubbing steel surfaces, Wear 262 (2007) 36-41

[22] C. Colombie, Y. Berthier, A. Floquet, L. Vincent, M. Godet, Fretting: load carrying capacity of wear debris, Journal of Tribology 106 (1984) 194

[23] M. Handbook, American Society for Metals, Metals Park, Ohio 44073 (1985)

[24] M.M. Haque, M.S.J. Hashmi, Investigation on microstructure of medium carbon steel deformed at strain rates of upto 105 per second at $-30^{\circ} \mathrm{C}$ to $235^{\circ} \mathrm{C}$, Journal of Materials Processing Technology 56 (1996) 119-125

[25] F.H. Stott, J. Glascott, G.C. Wood, Models for the generation of oxides during sliding wear, Proceedings of the Royal Society of London Series a-Mathematical Physical and Engineering Sciences 402 (1985) 167-186

[26] K. Hiratsuka, K. Muramoto, Role of wear particles in severe-mild wear transition, Wear 259 (2005) 467-476

[27] Q. Sunqing, Z. Zhongrong, D. Junxiu, C. Guoxu, Preparation of Ni Nanoparticles and Evaluation of Their Tribological Performance as Potential Additives in Oils, Journal of Tribology 123 (2001) 441-443

[28] S. Tarasov, A. Kolubaev, S. Belyaev, M. Lerner, F. Tepper, Study of friction reduction by nanocopper additives to motor oil, Wear 252 (2002) 63-69

[29] J. Zhou, Z. Wu, Z. Zhang, W. Liu, Q. Xue, Tribological behavior and lubricating mechanism of Cu nanoparticles in oil, Tribology Letters 8 (2000) 213-218

[30] H.L. Du, P.K. Datta, I.A. Inman, E. Kuzmann, K. Suvegh, T. Marek, A. Vertes, Investigations of microstructures and defect structures in wear affected region created on Nimonic 80A during high temperature wear, Tribology Letters 18 (2005) 395-404

[31] I.A. Inman, S. Datta, H.L. Du, J.S. Burnell-Gray, Q. Luo, Microscopy of glazed layers formed during high temperature sliding wear at 750 degrees C, Wear 254 (2003) 461-467

[32] J.C. Walker, I.M. Ross, C. Reinhard, W.M. Rainforth, P.E. Hovsepian, High temperature tribological performance of CrAlYN/CrN nanoscale multilayer coatings deposited on $\gamma$-TiAl, Wear 267 (2009) 965-975

[33] I.M. Hutchings, Tribology: friction and wear of engineering materials, Edward Arnold, 1992, 
[34] A. Neville, A. Morina, T. Haque, M. Voong, Compatibility between tribological surfaces and lubricant additives-How friction and wear reduction can be controlled by surface/lube synergies, Tribology International 40 (2007) 1680-1695 